\title{
Estudio preliminar de aminoaciduria en hepatitis infecciosa
}

Dres. Kenneth Jones*, Cecilia Escobar* y Marta Colombo** y seriorita Luz Arévalo, QF.***.

La hepatitis infecciosa aguda produce importantes alteraciones en la síntesis proteica y el catabolismo de aminoácidos a nivel del hígado. A esto hay que agregar el aumento de aminoaciduria que ocurre en los procesos de destrucción celular que acompañan las hepatitis graves. Quisimos saber cómo se traducen estas alteraciones fisiopatológicas en la aminoaciduria. La literatura es un poco ambigua; la mayoría de los autores sólo reconocen alteraciones de la aminoaciduria en hepatitis graves, insuficiencia hepática aguda o coma hepático. Davidsohn y Henry ${ }^{1}$ dicen categóricamente que en hepatitis aguda los valores de aminoaciduria son normales. Snydermun ${ }^{2-3}$ insiste que "las únicas alteraciones metabólicas que producen aminoaciduria generalizadas ocurren cuando hay una destrucción excesiva de tejidos como en el postoperatorio, en estados febriles agudos o en insuficiencia hepática aguda". Sin embargo, Hsia y Gellis", en un trabajo publicado en 1954, demuestran que en 18 casos de hepatitis infecciosa aguda estudiados, un tercio presentó moderada aminoaciduria con valores en $50 \%$ sobre lo normal, otro tercio presentó alteraciones límites y el último tercio era normal.

\footnotetext{
* Servicio de Pediatría, Hospital Carlos van Buren, Va!paraiso.

** Instituto de Nutrición y 'Tecnología de los Alimentos. Universidad de Chile, Sede Sur, Santiago.

***Laboratorio Clínico Hospital Carlos van Buren. Valparaíso.
}

Una revisión de la literatura mundial de los últimos seis años solicitada a través de BIREME no reveló ninguna nueva investigación en relación a este tema.

Por este motivo se realizó el presente estudio preliminar para comprobar si efectivamente había una alteración de la aminoaciduria en la hepatitis aguda benigna.

\section{OBJETIVOS}

1. Estudiar si en la hepatitis infecciosa aguda hay un aumento de excreción de aminoácidos por la orina.

2. Estudiar el pattern de la aminoaciduria en la hepatitis infecciosa.

\section{MATERIAL Y METODO}

Se estudiaron, entre junio y septiembre de 1976 , 12 niños con hepatitis infecciosa aguda de evolución benigna.

El diagnóstico de hepatitis infecciosa se hizo por los antecedentes, el examen físico y los exámenes de laboratorio de rutina (bilirrubinemia, pruebas de floculación y transaminasas). Solamente 1 niño tenía antecedentes de una inyección (vacuna) en los 6 meses previos.

Las edades fluctuaron entre los 18 meses y los 12 años. Se practicaron 18 exámenes de aminoaciduria, mediante un examen aislado de orina durante la fase de enfermedad aguda. 
La técnica usada era de cromatografía as. cendente en papel descrito por nosotros en un trabajo anterior ${ }^{5}$.

Los resultados expresados en micromoles/ día se compararon con las cifras de aminoacidurias obtenidas por nosotros en un trabajo en población de niños normales y de edad comparativa descrito en el mismo trabajo anterior ${ }^{5}$.

\section{RESULTADOS Y COMENTARIOS}

Los resultados generales se expresan en el cuadro 1 .

Cuadro 1

AMINOACIDURIA EN 12 NIÑOS CON HEPATITIS INFECCIOSA EXPRESADO ENMICROMOLES/DIA VALPARAISO, CHILE, 1976

\begin{tabular}{|l|r|r|r|r|r|r|r|r|r|r|r|r|r|r|}
\hline & Leu. & Fa. & Val. & Tir. & Prol. & Al. & Treo. & Glic. & Ser. & Glut. & Lis. & Hist. & Cist. \\
\hline J.I. & 5 años & & & & & & 456 & 682 & 4.330 & 3.080 & 139 & 555 & 263 & 1.360 \\
\hline S.P. & $21 / 2$ años & & & & & & 194 & 146 & 455 & 164 & 234 & 118 & 110 & 71 \\
\hline A.H. & 7 años & 196 & 156 & 220 & & & 1.160 & 433 & 5.500 & 980 & 353 & 2.830 & 2.670 & \\
\hline A.A. & 3 años & & & 146 & & & 190 & 142 & 915 & 653 & 234 & 233 & 110 & 70 \\
\hline & & 131 & 104 & 293 & 95 & & 770 & 288 & 915 & 653 & 472 & 1.880 & 222 & 1.140 \\
\hline J.G. & 8 años & & & & & & 385 & 288 & 1.880 & 1.330 & 465 & 960 & 900 & 143 \\
& & & & & & & 385 & 288 & 5.250 & 1.870 & 960 & 960 & 438 & 2.280 \\
\hline V.R. & 7 años & & & & & & 284 & 215 & 343 & 244 & & 350 & 160 & \\
\hline R.S. & 11 años & & & & & & & 288 & 910 & 648 & 236 & 468 & 221 & 284 \\
& & & & & & & & 288 & 910 & 648 & 469 & 469 & 221 & 284 \\
\hline H.Q. & $91 / 2$ años & & & & & & 385 & & 1.880 & 330 & & 469 & 440 & 284 \\
& & & & & & & 765 & 573 & 910 & 650 & & 235 & & 143 \\
\hline M.U. & 1 1/2 años & 132 & 105 & 147 & 109 & & 385 & 288 & 3.670 & 651 & 470 & 470 & 221 & 285 \\
\hline L.N. & 8 años & & & & & & 385 & 288 & 1.870 & 1.330 & 470 & 470 & 440 & 143 \\
& & & & & & & & 288 & 920 & 650 & 470 & 470 & 440 & 143 \\
\hline J.O. & 5 años & & & & & & 290 & 218 & 686 & 490 & 353 & 353 & 322 & \\
\hline M.V. & 12 años & & & & & & 385 & 475 & 7.350 & 5.210 & 235 & 599 & 363 & 363 \\
\hline
\end{tabular}

NOTA: En 6 niños se repitió el examen durante la fase aguda.

De los 12 niños estudiados solamente 2 $(16,7 \%)$ presentaron aminoaciduria normal. Los 10 restantes $(83,3 \%)$ presentaron aumento de la excreción de todos los aminoácidos estudiados comparado con niños normales de la misma edad. Los resultados expresados en promedio del grupo control y promedio de los 10 casos de hepatitis con aminoaciduria alterado se expresan en el gráfico 1.

El aumento de alanina ( $68 \%$ sobre la cifra promedio normal), treonina (31\%) y glutamina $(50 \%)$ fue moderado. El aumento de lisina (313\%), histidina (228\%) y cistina (187\%) era notorio. Pero lo que más llamó la atención era el enorme aumento de la glicina $(748 \%)$ y de la serina (516\%). En dos casos la eliminación urinaria de glicina sobrepasó los 5.000 micromoles/dia (promedio normal 350 micromoles/día). Hsia y Gellis ${ }^{4}$ hablan de un aumento moderado en un tercio de sus casos con cifras que llegan hasta $100 \%$ sobre lo normal.

Presentamos este estudio preliminar para volver a despertar el interés en un método de estudio que aparentemente ha sido abandonado en la hepatitis infecciosa y cuya importancia en la clínica de esta enfermedad no ha sido bien avaluado según la bibliografía a nuestro alcance. 


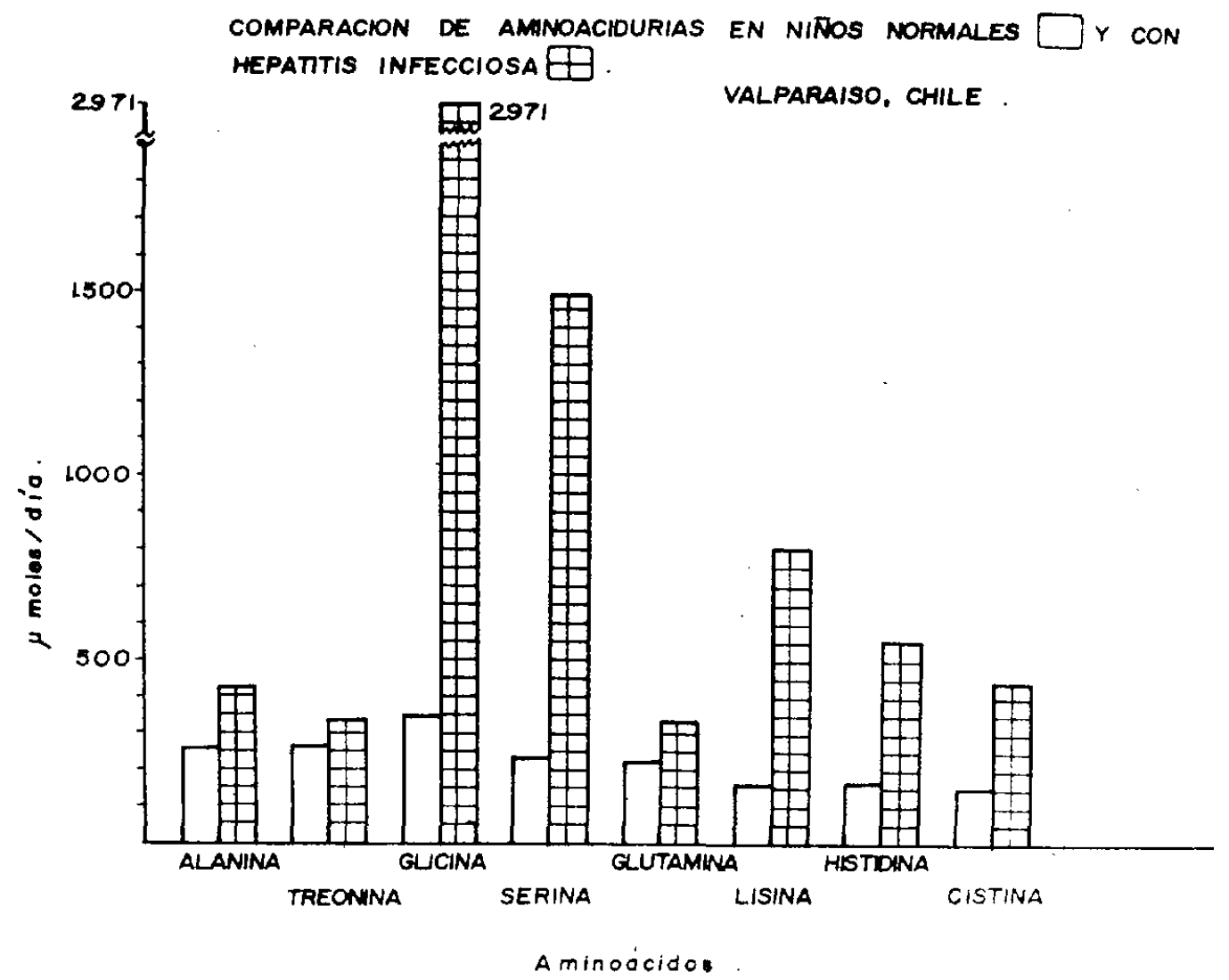

\section{CONCLUSIONES}

1. En la hepatitis infecciosa aguda hay una aminoaciduria generalizada.

2. El pattern de la aminoaciduria demuestra aumento moderado de alanina, treonina y glutamina. Aumento importante de la lisina, histidina y cistina. $Y$ un aumento muy exagerado de glicina y serina.

3. Es necesario mayor estudio para evaluar si estos hallazgos tienen aplicación útil en la clínica.

\section{RESUMEN}

1. Se presenta un estudio prelıminar de la aminoaciduria en 12 niños con hepatitis infecciosa aguda benigna entre 18 meses y 12 años de edad. Se usa el método de la cromotografia ascendente en papel.

2. Sotamente 2 niños presentan una aminoaciduria dentro de límites normales. Los 10 restantes tienen una aminoaciduria generalizada.

3. En los 10 niños con aminoaciduria anormal, la alanina, treonina y glutamina demuestran aumento moderado. Lisina, histidina y cistina presentan aumentos considerables. Glicina y serina aumentos muy exagerados.

\section{SUMMARY}

1. A preliminary study of aminoaciduria by ascending paper chromatography in 12 children with benign acute infectious hepatitis aged 18 months to 12 years in reported.

2. Only 2 children presented aminoaciduria within normal limits. The remaining 10 all presented a generalized aminoaciduria.

3. In the 10 cases with abnormal aminoaciduria alanine, threonine and glutamine were moderately increased. Lysine, histidine and cistine were markedley increased While glycine and serine were grossly increased.

\section{BIBLIOGRAFIA}

Dovidsohn and Henry. Clinical diagnosis by laboratory methods, W.B. Saunder Company, 1969.

* Snyderman, S.E. Ped. Clin. of N.A.. Feb. 1971 199.

3 Snyderman, S.E. and Holt, L.E. Advances in Pediatrics. Vol. 11:209. 1960 .

4 Hsta, D.Y.Y and Gelles, S.S. J. Clin. Investigation, 33: $1603,1954$.

- Escobar, C.: Colombo, . M.; Jones, K. and Arézalu, L. Aminoaciduria en población infantil normal de 0 a 6 años. Presentado a las VI Jornadas de Pediatría. Iiña del Mar, 1976. 